\title{
EFFECT OF ELASTIC DRIVING FORCE ON THE EVOLUTION OF MICROSTRUCTURES IN THE SECONDARY CREEP STAGE
}

\author{
Katsushi Tanaka ${ }^{1, a}$, Wataro Hashimoto ${ }^{1, b}$, Toru Inoue ${ }^{1, c}$ and Haruyuki Inui ${ }^{1, d}$ \\ ${ }^{1}$ Department of Materials Science and Engineering, Kyoto University, Sakyo-ku, Kyoto, Japan \\ ak.tanaka@materials.mbox.media.kyoto-u.ac.jp, \\ bwataro-hashimoto@student.mbox.media.kyoto-u.ac.jp, ${ }^{\mathrm{c}}$ imouetoro@gmail.com, \\ dharuyuki-inui@mtl.kyoto-u.ac.jp
}

Key words: raft structure, creep, elastic properties, XRD.

\begin{abstract}
The effect of elastic driving force on the microstructural change of superalloys in the secondary creep stage is evaluated by elastic energy calculations with the concept of effective eigen strain where both lattice mismatch and creep strain are taken into account The elastic energy calculations indicates that the elastic state in the secondary creep stage is totally different to that in the initial one where the lattice misfit between $\gamma$ and $\gamma^{\prime}$ phases is over accommodated along the [100] and [010] directions by creep deformation in the $\gamma$ phase. The excess creep dislocations for the over accommodation are required so as to develop an internal stress field to prevent further creep deformations. The planer raft structure with the plane normal oriented to the [001] direction is unstable in the over accommodated state. The $\gamma / \gamma^{\prime}$ lamellar interfaces will be inclined to make a wavy raft structure of which elastic energy is lower than the ideal 001 planer raft structure.
\end{abstract}

\section{Introduction}

The efficiency of aircraft engines and gas turbine generators is improved by increase in the gas temperature and consequently the operating temperature of the turbine blades equipped. Since nickel-base single crystal superalloys exhibit superior high-temperature creep strength and oxidation resistance, they have been applied to turbine blades in industrial gas turbines and aero-engines. However, creep strength of the blades is gradually degraded in service. It is known that superior performances of superalloys and their degradation are closely related to the change in its microstructure as indicated in Fig. 1. The initial microstructure of the alloys is called as cuboidal structure, where cuboidal precipitates of the $\gamma^{\prime}$ phase (the $\mathrm{L}_{2}$ ordered structure) are aligned like a cubic lattice in the $\gamma$ matrix (the fcc disordered structure). When superalloys are subjected to creep at high-temperatures under a relatively low tensile stress along the [001] direction, $\gamma^{\prime}$ cuboids are coarsened normal to the stress direction to form the "raft structure", where $\gamma$ and $\gamma^{\prime}$ plates expand in the (001) plane stack alternately. Since the dislocation motion is hindered by lateral $\gamma / \gamma^{\prime}$ lamellar interfaces, creep deformation is suppressed significantly by the formation of the microstructure $[1,2]$. Under the influence of applied stress, the lamellar interfaces in the raft structure gradually deviate from the (001) plane so as to form a wavy raft structure in the secondary creep stage. The amplitude of the wave gradually increases as proceeding creep deformations [3,4]. Since the inclination is considered to be closely related to the degradation of creep strength, the origin of the local incline of $\gamma / \gamma^{\prime}$ interfaces observed in the secondary creep stage is an important factor for understanding the collapse mechanism of the raft structure.

The role of elastic driving force to form the raft structure in the primary creep stage has extensively been discussed [5-9]. From their previous works, it is known that an applied external stress and introductions of creep dislocations slightly modify the internal elastic fields in an early stage of the primary creep, and the elastic fields drive the initial cuboidal structure to the raft structure. On the other hand, our previous experiments indicate that the internal elastic fields are continuously modified by creep even after the raft structure forming [4], and the role of such extensively modified elastic 


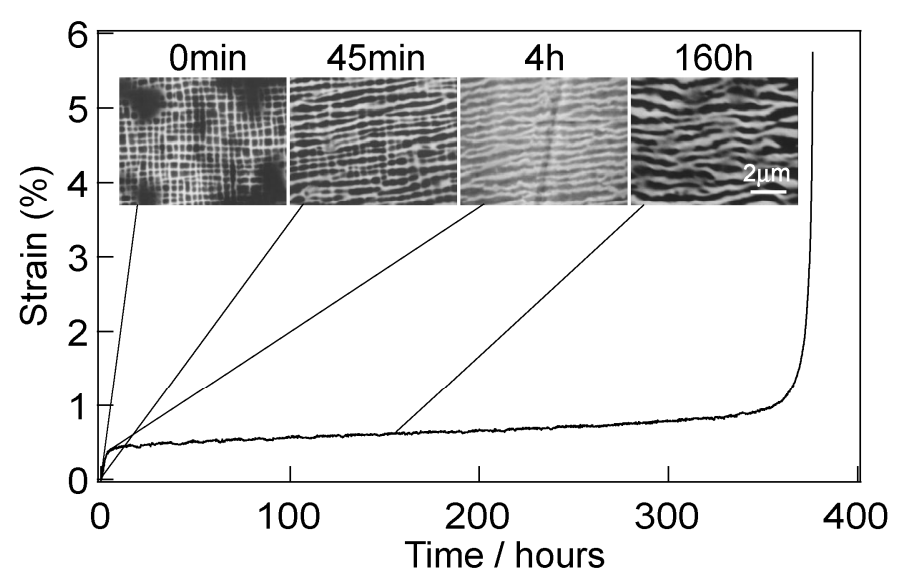

Fig. 1. Typical creep curve of Ni-base superalloy. The alloy is TMS-26 and the condition of creep deformation is tensile stress of $137 \mathrm{MPa}$ at $1373 \mathrm{~K}$. Inset SEM micrographs show the microstructural change through creep deformation.

fields is still an open question. In this report, the effect of elastic driving force on the evolution of microstructures after forming the raft structure is discussed from elastic energy calculations.

\section{Method of calculation}

The microstructural evolution without chemical composition changes is determined by the change in the total mechanical energy. The total mechanical energy corresponding to the Gibb's free energy of the system, $U$, is expressed as

$$
U=U_{\text {int }}+U_{\text {ext }}+U_{\text {pot }}
$$

where $U_{\text {int }}, U_{\text {ext }}$ and $U_{\text {pot }}$ are the internal elastic energy, elastic energy caused by the application of an external stress and the potential energy of an external stress, respectively. In the following calculations, we assume that the elastic constants of the $\gamma$ and $\gamma^{\prime}$ phases are identical with each other. On the assumption, only $U_{\text {int }}$ is affected by the morphology of the microstructure. In other words, the internal energy is not affected by external stresses and/or strains [10].

From the reported results of experiments and theoretical calculations, creep dislocations in modern superalloys are considered to move only in $\gamma$ channels perpendicular to the applied stress but not to pass through $\gamma$ channels parallel to the applied stress and $\gamma^{\prime}$ precipitates under a typical tensile creep condition, for example, at $1373 \mathrm{~K}$ under $137 \mathrm{MPa}$ [11]. As a consequence, only (or mainly) the $\gamma$ phase is plastically deformed by the motion of creep dislocations. Furthermore, creep dislocations are almost homogeneously introduced in the lateral $\gamma$ channels [12]. With these knowledge, we have introduced the "effective eigenstrain" of the $\gamma$ phase, $\varepsilon^{*}{ }_{\text {eff }}$, which is the sum of the lattice misfit between $\gamma$ and $\gamma^{\prime}$ phases, $\varepsilon_{0}$, and the amount of plastic strain caused by the introduction of creep deformations, $\varepsilon_{\mathrm{p}}$. The lattice misfit is defined as $\varepsilon_{0}=\left(a_{\gamma}-a_{\gamma^{\prime}}\right) / a_{\gamma^{\prime}}$ where $a$ denotes the lattice parameter. When we impose the restriction on the tensile stress direction to be exact [001], all the eight of $<101>\{111\}$ slip systems are expected to operate equivalently; the plastic deformation can be treated as a simple tetragonal distortion. The effective eigenstrain of the $\gamma$ phase with respect to the $\gamma^{\prime}$ phase is expressed as,

$$
\varepsilon_{\mathrm{eff}, 11}^{*}=\varepsilon_{\mathrm{eff}, 22}^{*}=\varepsilon_{0}-\frac{a_{\gamma}}{a_{\gamma^{\prime}}} \frac{\varepsilon_{\mathrm{p}}}{2}, \varepsilon_{\mathrm{eff}, 33}^{*}=\varepsilon_{0}+\frac{a_{\gamma}}{a_{\gamma^{\prime}}} \varepsilon_{\mathrm{p}} \text { and } \varepsilon_{\mathrm{eff}, i j}^{*}=0(i \neq j) .
$$

Since the lattice constants of the $\gamma$ and $\gamma^{\prime}$ phases have a similar value, the factor $a_{\gamma} / a_{\gamma^{\prime}}$ is closed to unity and we ignore the factor in the following calculations. By using this effective eigenstrain, one can estimate the variation of the internal stress/strain fields and the internal elastic energy with creep deformation.

The internal elastic strain field for a given effective eigen strain has been calculated by means of the Fourier-transformation method proposed by Khachaturyan [13], in which the elastic strain at position $\boldsymbol{r}, \varepsilon(\boldsymbol{r})$, is calculated as 
a)

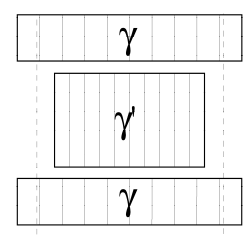

b)
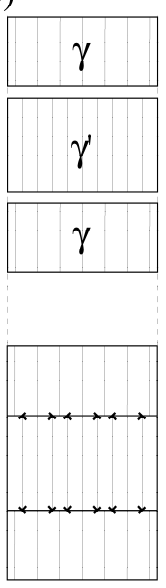

c)
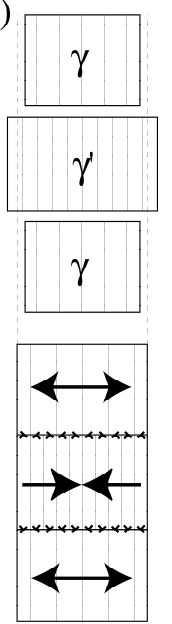

Fig 2. Schematic illustration of the internal-stress evolution with an increase in creep deformations of the $\gamma$ phase. Upper and lower figures are unstressed state and semi-coherent state at each stage of creep deformations, respectively. Arrows indicate the force directions due to the internal stress field. The vertical lines indicate a fixed number of the (100) lattice planes of the $\gamma$ and $\gamma^{\prime}$ phases. a), initial state (completely coherent), b), the lattice mismatch is fully relaxed and c), excess creep dislocations are introduced.

$$
\varepsilon_{m n}(\boldsymbol{r})=\frac{1}{2} \int_{-\infty}^{\infty}\left\{C_{i j k l} \bar{\xi}_{l}\left(G_{i m}^{-1} \bar{\xi}_{n}+G_{i n}^{-1} \bar{\xi}_{m}\right) \hat{\varepsilon}_{k l}^{*}(\xi)-\hat{\varepsilon}_{m n}^{*}(\xi)\right\} \exp (i \xi \cdot \boldsymbol{r}) d \xi
$$

where $C_{i j k l}$ is the elastic stiffness constants, $\hat{\varepsilon}_{k l}^{*}(\boldsymbol{\xi})$ is the Fourier-transformed effective eigenstrain and $G_{i m}$ is the Green's function given by

$$
G_{i m}=C_{i p m q} \bar{\xi}_{p} \bar{\xi}_{q} \text {, where } \bar{\xi} \text { is the unit vector parallel to } \xi \text {. }
$$

The internal elastic stress, $\sigma_{i j}$, and energy, $U_{i n t}$, are respectively given by

$$
\sigma_{i j}=C_{i k j l} \varepsilon_{k l} \text { and } U_{\text {int }}=\frac{1}{2} \sigma_{i j} \varepsilon_{i j}
$$

The parameters used are reference to the experimental data of the TMS-26 alloy. The lattice mismatch, $\varepsilon_{0}$, is set to be $0.2 \%$, and the elastic constants are $C_{11}=210, C_{12}=150$ and $C_{44}=95 \mathrm{GPa}$, respectively, which were experimentally measured at $1373 \mathrm{~K}$ [14]. The volume fraction of the $\gamma$ phase $\left(f_{\gamma}\right)$ is set to be $44 \%$, referring to the value experimentally determined by the area fractions of the $\gamma$ and $\gamma^{\prime}$ phases in SEM micrographs. According to our previous experiments, the magnitude of creep deformations in the $\gamma$ phase, $\varepsilon_{\mathrm{p}}$, is set to the value from 0 to $0.8 \%$. Note that the value of $0.4 \%$ corresponds to the value for fully relaxing the lattice mismatch along the [100] and [010] directions (see equation (2)). The sign of internal stress along the [100] and [010] directions is changed at the value of $\varepsilon_{\mathrm{p}}$ being $0.4 \%$ as illustrated in Fig. 2 .

\section{Results and Discussion}

The model for the elastic-plastic calculation is illustrated in Fig. 3(a), where the lamellae of $\gamma$ and $\gamma^{\prime}$ phases ideally spread but the orientation of the plane is inclined from (001). Figures 3(b)-(f) show equi-energy contours of the internal elastic energies $U_{\text {int }}$ calculated as a function of crystallographic orientation of the lamellae normal for different magnitude of the creep strain in the $\gamma$ phase. For $\varepsilon_{\mathrm{p}}=$ $0 \%$ (Fig. 3(b)), the minimum of the internal elastic energy is achieved when the lamellae normal is directed to [001] or [100]. As seen in Figs. 3(c)-(d), the energy at [001] decreases with the increase in $\varepsilon_{\mathrm{p}}$, while the energy at [100] increases. This agrees with the previous works, that is the $001 \mathrm{raft}$ structure being elastically stabilized by introductions of creep dislocations [14]. However, when the creep strain, $\varepsilon_{\mathrm{p}}$, is larger than $0.4 \%$ (Figs. 3(e)-(f)), the [001] direction is not the energy minimum direction any more but forms a local maximum. 
a)

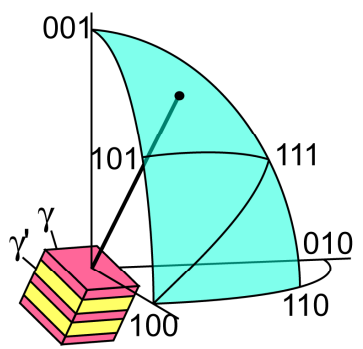

d)

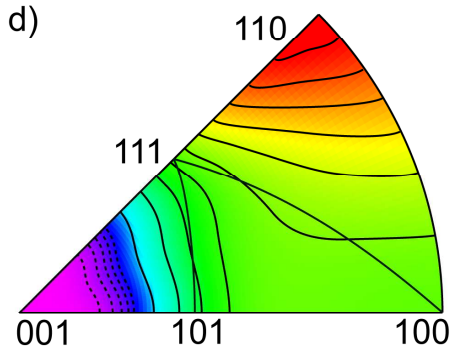

b)

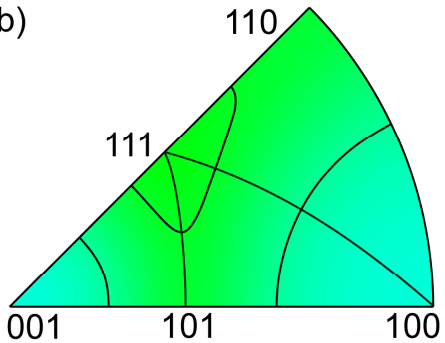

e)

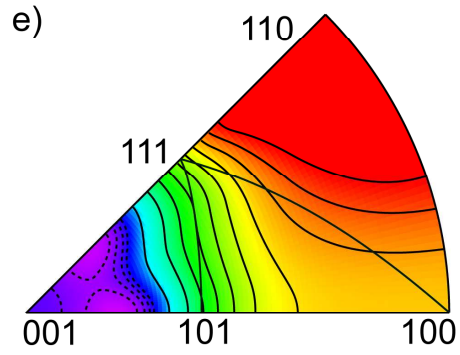

c)

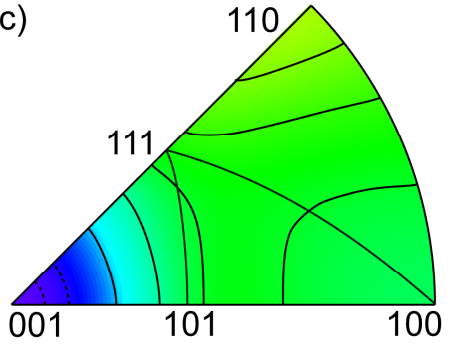

f)

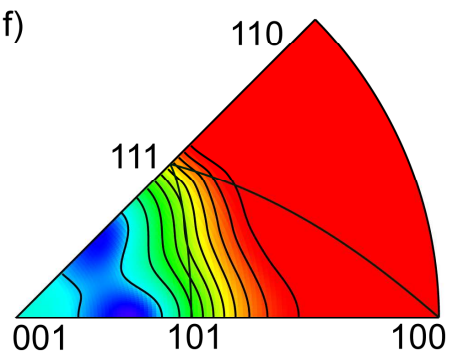

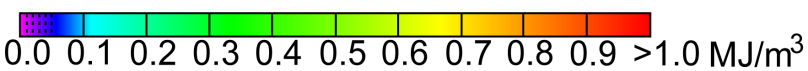

Fig 3. Equi-energy contours of elastic energy as a function of lamellar plane normal direction plotted on the standard stereo projection. a), model of the calculation, b), $\left.\varepsilon_{\mathrm{p}}=0 \%, \mathrm{c}\right), \varepsilon_{\mathrm{p}}=0.2 \%, \mathrm{~d}$ ), $\left.\varepsilon_{\mathrm{p}}=0.4 \%, \mathrm{e}\right), \varepsilon_{\mathrm{p}}=0.6 \%$ and f), $\varepsilon_{\mathrm{p}}=0.8 \%$.

If the magnitude of creep strain in the $\gamma$ phase does not reach the value of $0.4 \%$, the 001 raft structure is elastically stable. Since the introduction of creep dislocations are governed by the stress field in the $\gamma$ phase, the Peatch-Koeloer force applied to the dislocations is considered. The internal elastic energy $\left(U_{\text {int }}\right)$, total mechanical energy $(U)$ and the maximum shear stress applied to dislocations with the Burgers vector of $(1 / 2)<101>$ in the $\gamma$ phase $\left(\sigma_{\max }\right)$ calculated for the ideal 001 raft and an external tensile stress of $137 \mathrm{MPa}$ are shown in Figure 4 as a function of creep strain of the $\gamma$ phase $\left(\varepsilon_{\mathrm{p}}\right)$. Of interest to note is that creep dislocations loose their driving force for their motion not at $\varepsilon_{\mathrm{p}}=0.4 \%$ but at $\varepsilon_{\mathrm{p}}=0.74 \%$, because the stress tensor in the $\gamma$ phase for $\varepsilon_{\mathrm{p}}=0.4$ and $0.74 \%$ are respectively calculated as,

$$
\left.\sigma\right|_{\varepsilon_{\mathrm{p}}=0.4 \%}=\left(\begin{array}{ccc}
0 & 0 & 0 \\
0 & 0 & 0 \\
0 & 0 & 137 \mathrm{MPa}
\end{array}\right) \text { and }\left.\sigma\right|_{\varepsilon_{\mathrm{p}}=0.74 \%}=\left(\begin{array}{ccc}
137 \mathrm{MPa} & 0 & 0 \\
0 & 137 \mathrm{MPa} & 0 \\
0 & 0 & 137 \mathrm{MPa}
\end{array}\right) \text {, }
$$

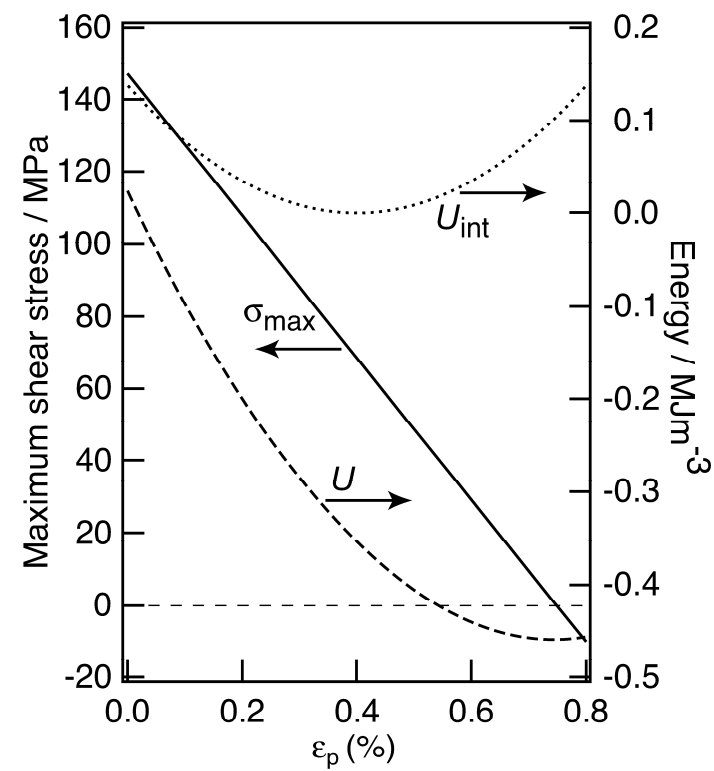

Fig. 4. Maximum shear stress applied to creep dislocations ( $\sigma_{\max }$ : solid lines), internal elastic energy ( $U_{\text {int }}$ : dotted lines) and total mechanical energy $(U$ : dashed lines) as a function of creep strain of the $\gamma$ phase $\left(\varepsilon_{\mathrm{p}}\right)$. The calculation assumes the perfect 001 raft structure. Total energy minimum is realized at about $\varepsilon_{\mathrm{p}} \sim 0.74 \%$, at which the shear stress for creep vanishes but the internal elastic energy is not minimized and the 001 raft structure is elastically unstable (see text). 
where tensile stress is indicated by positive value. The former indicates that the lattice mismatch is fully relaxed and an external stress is directly applied to the $\gamma$ phase. The latter indicates that the internal stresses along the [100] and [010] directions are added to an external applied stress, which makes the stress field to a negative hydrostatic pressure. When the volume fraction of the $\gamma$ phase of $44 \%$ is taken into account, the creep strain of the $\gamma$ phase of $\varepsilon_{\mathrm{p}}=0.74 \%$ corresponds to the total strain of $\varepsilon_{\text {tot }} \sim 0.32 \%$ of the whole material. The total strain actually agrees with the experimental creep strain at the beginning of the secondary creep stage; see Fig. 1. This indicates that the 001 raft structure is already elastically unstable at the beginning of the secondary creep stage.

In order to know more realistic microstructural change caused by the elastic instability, three types of microstructural change with inclination of $\gamma / \gamma^{\prime}$ lamellar interfaces are considered as shown in Fig. 5. The first one is a $\gamma^{\prime}$ lamellar simply waving without changing in its thickness. Second and third ones

(a)

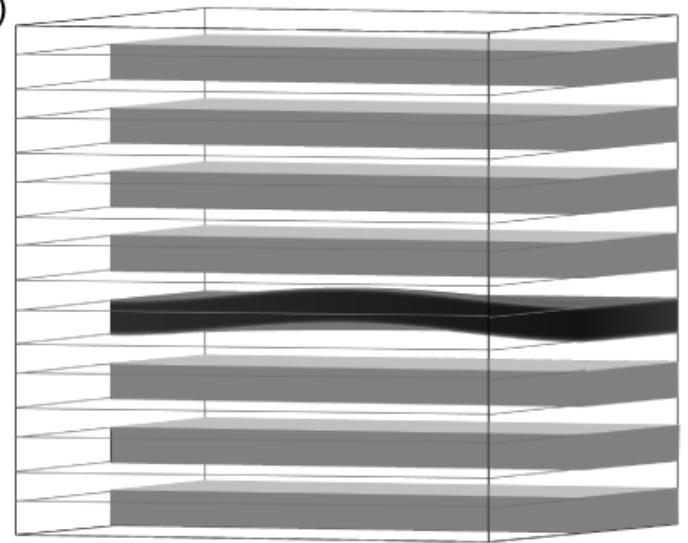

(b)

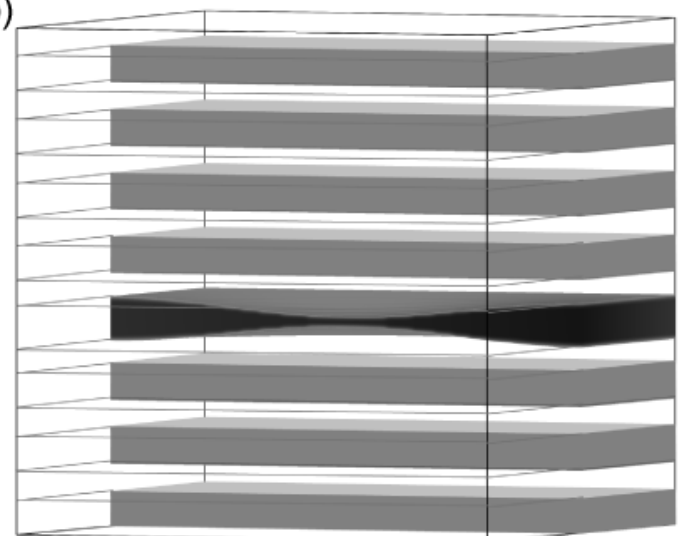

(c)

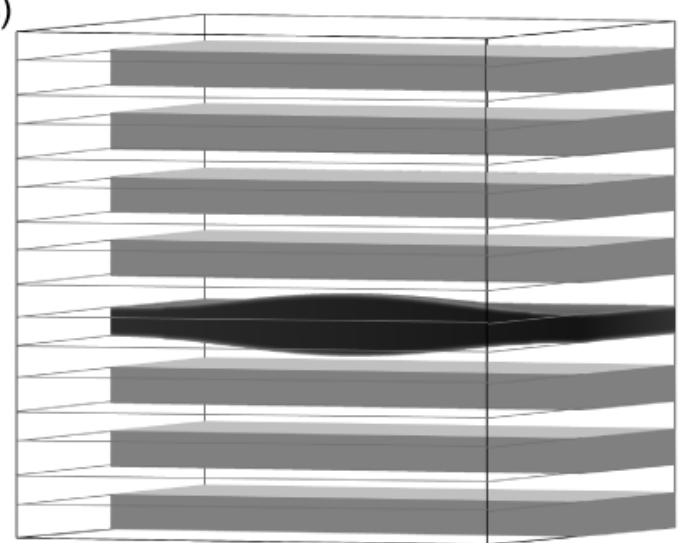

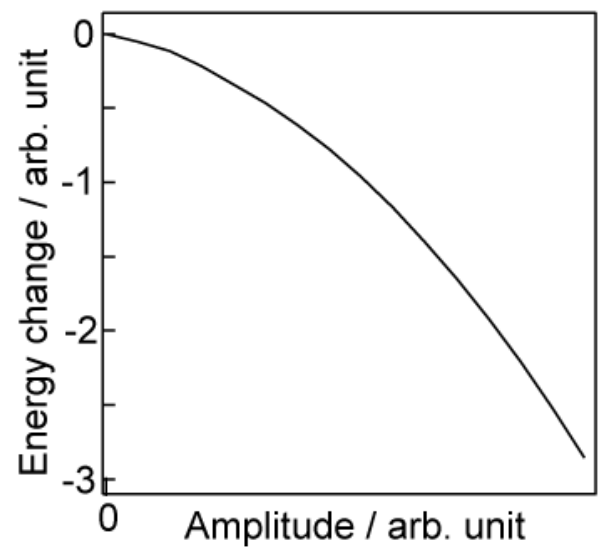
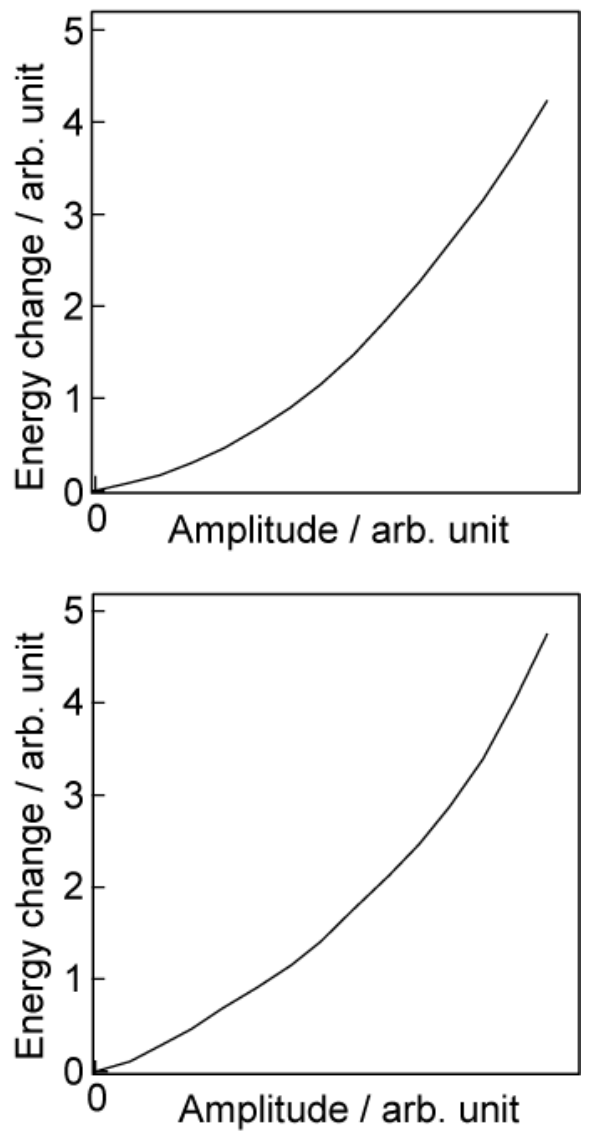

Fig. 5. Variation of elastic energies with inclination of $\gamma / \gamma^{\prime}$ lamellar interfaces. (a) A part of $\gamma^{\prime}$ lamellar waves, (b) the thickness of a part of $\gamma^{\prime}$ lamellar reduces and (c) the thickness of a part of $\gamma^{\prime}$ lamellar increases, respectively. All the calculations are carried out under the condition of $f_{\gamma}=$ $44 \%$ and $\varepsilon_{\mathrm{p}}=0.74 \%$. 
simulate pinching off and swelling out of a $\gamma^{\prime}$ lamellar, where the thickness of a part of $\gamma^{\prime}$ lamellar changes. The variations of elastic energy caused by the morphology changes are calculated and plotted as a function of the amplitude of the change. When a part of $\gamma^{\prime}$ lamellar waves, elastic energy decreases with increasing the amplitude of the waving as shown in Fig. 5(a). On the other hand, as shown in Figs. 5(b) and 5(c), elastic energy increases when the thickness of a part of $\gamma^{\prime}$ lamellar changes. This fact well agree with the microstructural evolution observed in the secondary creep stage where the amplitude of the waving of $\gamma^{\prime}$ lamellae gradually increases with increasing creep time but $\gamma^{\prime}$ lamellae are not pinched off and swelled out.

\section{Summary}

From the present strain-energy calculation based on the concept of effective eigenstrain, we have found that creep strain stabilizes the 001 raft structure if the amount is smaller than that required for fully relaxing the lattice mismatch, but they deteriorate the elastic stability of the raft structure when the creep strain becomes larger. Because of the existence of the external tensile stress, the number of creep dislocations inevitably introduced to suppress the further creep deformation is more than that needed for accommodation of the lattice mismatch. As a result, the 001 raft structure changes its morphology to wavy ones in the secondary creep stage.

\section{Acknowledgments}

A part of this work was supported by Grant-in-Aid for Scientific Research (B) from the Ministry of Education, Culture, Sports, Science and Technology (MEXT), Japan and and in part by the Global COE (Center of Excellence) Program of International Center for Integrated Research and Advanced Education in Materials Science from the MEXT, Japan.

\section{References}

[1] J.K. Tien and R.P. Gramble: Metall. Trans. Vol. 3 (1972), p.2157.

[2] D.D. Pearson, F.D. Lemkey and B.H. Kear: Superalloys 1980 (The Minerals, Metals \& Materials Society 1980), p. 513.

[3] A. Epishin, T. Link, P.D. Portella and U. Brückner: Acta Mater., Vol. 48 (2000), p. 4169.

[4] K. Tanaka, T. Kajikawa, T. Ichitsubo, M. Osawa, T. Yokokawa and H. Harada, Mater. Sci. Forum, Vol. 475-479(2005), p. 619.

[5] S. Socrate and D. M. Parks: Acta Metall. Mater., Vol. 41 (1993), p. 2185.

[6] J.Y. Buffiere and M. Ignat: Acta Metall. Mater., Vol. 43 (1995), p. 1791.

[7] F.R.N. Nabarro, C.M. Cress and P. Kotschy: Acta Mater., Vol. 44 (1996), p. 3189.

[8] N. Matan, D.C. Cox, C.M.F. Rae, R.C. Read: Acta Mater., Vol. 47 (1999), p. 2031.

[9] T. Ichitsubo and K. Tanaka: Acta Mater., Vol. 53 (2005), p. 4497.

[10] G. Colonnetti: Atti Accard Naz Lincei Rc, Vol. 24 (1915), p.404.

[11]L. Müller, U. Glatzel and M. Feller-Kniepmeier: Acta Metall. Mater., Vol. 41 (1993), p. 3401.

[12] T.M. Pollock and A.S. Argon: Acta Metall. Mater., Vol. 40 (1992), p. 1.

[13] A.G. Khachaturyan: Theory of Structural Transformation in Solids, (Wiley, New York, 1983).

[14] T. Ichitsubo, D. Koumoto, M. Hirao, K. Tanaka, M. Osawa, T, Yokokawa and H. Harada: Acta Mater., Vol. 51 (2003), p. 4863. 